\title{
Linguistique française et cognition
}

\author{
Catherine Fuchs \\ LATTICE, CNRS/ENS
}

\section{Introduction}

La question des liens entre linguistique française et cognition peut se décliner de plusieurs façons. Si l'on entend par 'linguistique française' l'ensemble des travaux de linguistique portant sur le français, quelle que soient les théories sur lesquelles ils prennent appui, la question revient à identifier parmi ces théories celles qui se déclarent cognitives. Dans cette perspective, rien ne concerne spécifiquement la linguistique française : au même titre que les spécialistes de n'importe quelle autre langue, les francisants soucieux de cognition se retrouvent au sein des associations (nationales ou européenne) de linguistique cognitive.

En revanche, la question prend une autre dimension si l'on s'attache à certains édifices théoriques élaborés par des linguistes français ou francophones (et largement repris dans des travaux de linguistique française) - ceci bien évidemment sans chauvinisme aucun, et en conservant à l'esprit que la science a vocation à dépasser toutes les frontières. C'est cette seconde perspective que j'adopterai ici.

Je rappellerai tout d'abord ( $§ 1$ ) les conditions historiques d'émergence, aux Etats-Unis, de la linguistique dite 'cognitive', qui s'est construite sans référence aucune aux théories de linguistique française développées (antérieurement ou parallèlement) en Europe. Certaines de ces théories, pourtant, proposaient des approches qui, en droit, participent d'une problématique cognitive; je m'attacherai à deux grands courants de linguistique française tout à fait représentatifs à cet égard : la théorie psychomécanique de Gustave Guillaume ( $\$ 2$ ) et les théories de l'énonciation (§ 3).

\section{La linguistique dite 'cognitive'}

Dans un article récent, fort stimulant, Gilbert Lazard affirme — c'est le titre même de son papier — que "la linguistique cognitive n'existe pas" (Lazard, 2007). Résumons tout d'abord l'argumentation de l'auteur, avant de rappeler le contexte historique qui a présidé à l'émergence de théories linguistiques dites 'cognitives', et d'évoquer les principaux enjeux de ce tournant pour la discipline.

\subsection{La linguistique cognitive existe-t-elle ?}

Selon notre auteur, le terme de 'sciences cognitives' désigne un ensemble de disciplines qui, telles la neurobiologie, la psychologie, ou l'intelligence artificielle, "prennent pour objet des aspects divers de l'activité sensorielle et intellectuelle par laquelle l'être humain prend connaissance du monde qui l'entoure" (Lazard, art. cit. : 3). Or, dit-il, si l'on inclut la linguistique dans cet ensemble, au nom des liens entre le langage et la pensée, alors "toute linguistique est cognitive". A l'inverse, si on l'en exclut, au nom de la spécificité des phénomènes langagiers et qu'on la considère comme une discipline connexe mais distincte, alors “aucune linguistique n'est cognitive". Dans un cas comme dans l'autre, "la notion de linguistique cognitive est obscure" (ibidem).

Les théories linguistiques se proclamant cognitives seraient toutes, en effet, confrontées au dilemme suivant. Soit elles ne feraient que revenir à la conception traditionnelle de la langue comme système symbolique de mise en correspondance entre formes et sens, soit elles sortiraient du champ propre de la discipline, en tentant de trouver des motivations 'externes' aux phénomènes linguistiques observés ou d'inférer des propriétés générales de l'esprit humain à partir de ces observations. Dans le premier cas, ce ne serait que de la linguistique (au sens le plus classique du terme); dans le second, ce ne serait plus de la linguistique. Quant aux 'instruments intellectuels' (comme, par exemple, la notion de prototype) que ces théories, soucieuses d'ouverture, peuvent se trouver emprunter à d'autres disciplines des sciences 
cognitives, “à vrai dire, pour ce faire il [= le linguiste $]$ n'a pas lieu, en principe, de se borner aux disciplines voisines : il peut prendre son bien partout où il le trouvera" (art. cit. : 15).

En définitive, l'appellation 'linguistique cognitive' ne serait donc qu'une "expression à la mode, dépourvue de sens ailleurs qu'aux Etats-Unis et en tout cas chez tous ceux qui n'ont pas subi l'emprise du générativisme. L'adjectif est de trop : la linguistique cognitive, c'est de la linguistique tout court. Cela dit, cette mode comporte un risque, celui de noyer le linguistique dans le cognitif, autrement dit d'oublier sa spécificité" (art. cit. : 14).

Pour en juger, tournons-nous donc vers ce que l'on est convenu d'appeler le 'tournant cognitif' de la linguistique, au milieu des années 1950 aux Etats-Unis (les repères historiques qui suivent sont repris de Fuchs, $2004: 6-12$ ).

\subsection{L'émergence des théories linguistiques dites 'cognitives'}

L'historiographie officielle s'accorde à faire remonter ce tournant aux deux conférences qui, en 1956, ont réuni, autour d'un projet épistémologique commun connu sous le nom de 'programme cognitiviste', le linguiste Noam Chomsky, le psychologue Herbert Simon et le spécialiste d'intelligence artificielle Marvin Minsky. L'objectif en était de caractériser le fonctionnement de l'esprit à travers les facultés qu'il développe (en particulier à travers la faculté de langage); et l'hypothèse fondatrice était que la cognition humaine pourrait être définie, à la manière d'une machine, en termes de calculs correspondant au traitement des divers types d'informations reçues par l'humain. C'est ainsi que la linguistique - en l'occurrence une certaine linguistique formelle - s'est trouvée, de fait, participer aux débuts de l'entreprise cognitiviste.

Le paradigme classique, qui s'est développé dans ce cadre, est appelé 'computo-représentationnel symbolique'. Il est fondé sur l'idée de calculs (ou 'computations') définis en termes d'opérations sur des 'symboles', lesquels auraient une réalité à la fois physique (ils seraient inscrits, d'une manière ou d'une autre, dans le cerveau) et sémantique (ils 'représenteraient' le monde objectif). L'activité de langage se ramènerait donc à un traitement d'informations mettant en jeu (niveau syntaxique) des règles de manipulation de symboles, c'est-à-dire d'éléments physiques (niveau neurobiologique) qui représenteraient adéquatement le monde réel (niveau sémantique). On notera au passage que ce cognitivisme de la fin des années 1950 se fondait largement sur la métaphore de 'l'esprit-machine' (partagée par la psychologie cognitive, la philosophie cognitive, et l'intelligence artificielle) : l'analogie avec le cerveau n'a été massivement exploitée que plus tard, vers la fin des années 1980, dans le cadre du rapprochement avec les neurosciences.

Ce paradigme classique est celui qui a été adopté, en linguistique, par la théorie chomskienne. Les principales caractéristiques en sont : une démarche hypothético-déductive, une perspective 'modulariste' (à la Fodor), une conception du langage comme instrument d'expression de la pensée permettant la transmission d'informations à propos du monde, et le recours à des modélisations de type logicoalgébrique. La linguistique chomskienne a donc été la première à se revendiquer explicitement comme une linguistique cognitive, en prônant une approche dite 'naturaliste' de l'objet langage. Pour reprendre les termes de Rouveret (2004: 30), "le langage humain est un objet 'psychologique', composante de l'esprit humain, physiquement représenté dans le cerveau, et faisant partie de l'équipement biologique de l'espèce, qui, précisément parce qu'il est ce type d'objet, peut et doit être analysé suivant les mêmes méthodes d'investigation que celles qui sont en œuvre dans les sciences de la nature".

Au fil des années, le paradigme cognitiviste des débuts a fait l'objet de divers types de critiques au sein de l'ensemble des sciences cognitives. Progressivement, des alternatives à l'orientation symbolique se sont fait jour, visant à définir un nouveau type de paradigme, parfois désigné sous le terme de 'constructivisme'. 
En écho à cette évolution générale, de nouveaux courants ont émergé, dès le début des années 1970, au sein de la linguistique dite cognitive, qui cherchaient à se démarquer — plus ou moins fortement selon les cas - du paradigme cognitiviste initial. C'est ainsi que, sur la côte Ouest des Etats-Unis, plusieurs auteurs venus de la grammaire générative (George Lakoff, Ronald Langacker, Leonard Talmy, Gilles Fauconnier) ont élaboré diverses formes de 'grammaires cognitives', en réaction contre l'option modulariste et la prééminence de la syntaxe prônées par les chomskiens. Les grammaires cognitives récusent le postulat selon lequel les grammaires formelles constitueraient des modèles adéquats de la cognition linguistique, et cherchent à relier les phénomènes langagiers aux processus généraux de la cognition (comme, par exemple, la perception — d'où l'importance accordée à l'espace). Par différence avec les grammaires formelles, cet autre courant de linguistique cognitive se caractérise par une démarche plus inductive et par une approche 'interactioniste' : une place centrale est accordée à la sémantique, réputée informer la syntaxe et le lexique avec lesquels elle interagit. La conception du langage y est davantage 'émergentiste' que représentationnelle: le langage est envisagé comme instrument de conceptualisation active du monde et/ou comme instrument de communication. Enfin, les outils de modélisation empruntent préférentiellement à la géométrie, aux systèmes dynamiques, ou au connexionnisme, plutôt qu'à l'algèbre et à la logique mathématique. Pour ces théories, le noyau dur de la langue ne réside pas dans les règles de la syntaxe mais dans les opérations de construction de la signification.

En bref, la linguistique dite cognitive, ainsi consignée par l'historiographie officielle, est bien née aux Etats-Unis et ce tournant théorique a effectivement débouché, s'agissant des grammaires cognitives, sur une remise en question des postulats du générativisme. Comme l'affirme Lazard, s'agit donc bien d'un mouvement initié outre-Atlantique et qui s'est développé sans lien avec les traditions de la vieille Europe (à l'exception du courant 'néo-fonctionnaliste' qui constitue un cas à part et mériterait, de ce point de vue, un développement spécifique). Mais, très vite, les nouveaux paradigmes instaurés par ce mouvement ont été adoptés par une partie des chercheurs européens, en linguistique française comme ailleurs.

Reste à présent à voir si la linguistique dite cognitive n'est, finalement, que "de la linguistique tout court", selon la formule de Lazard.

\subsection{Les enjeux d'une linguistique cognitive}

Rappelons que, parmi les sciences cognitives, la linguistique occupe une place à part : elle est la seule à avoir le langage pour objet d'étude exclusif, et elle aborde cet objet sous l'angle particulier de la diversité des langues. L'intérêt des linguistes pour des questions d'ordre cognitif (au sens large) ne date certes pas d'aujourd'hui : il existe en la matière une longue tradition, qui remonte à l'Antiquité, de réflexion sur les rapports entre les langues, la pensée, le raisonnement, l'action, etc. Pour autant, cette problématique générale et relativement diffuse ne se confond pas avec celle, plus circonscrite, d'une linguistique se voulant 'cognitive'.

La linguistique dite cognitive entend précisément ne pas se réduire à "de la linguistique tout court", car aux exigences classiques de toute théorie de linguistique générale, elle en ajoute une autre : la pertinence cognitive. Une théorie linguistique se voulant cognitive doit en effet pouvoir s'articuler de façon explicite avec des modèles généraux de l'architecture fonctionnelle de l'esprit et/ou de l'architecture neuronale du cerveau — d'où la question évoquée plus haut, de la 'naturalisation' de l'objet langage (Fuchs, 2004 : 1 6 ; Fuchs, $2007: 37-38$ ).

La perspective cognitive en linguistique conduit donc, non seulement à s'interroger sur l'ensemble des connaissances spécifiques que maîtrise l'esprit humain au travers des différents systèmes des langues, mais aussi à se demander comment ces connaissances sont organisées pour pouvoir être acquises et mises en œuvre dans l'activité de langage. D'où toute une série de questions qui concernent, par-delà l'architecture structurale des connaissances linguistiques, leur architecture fonctionnelle. Par exemple, sous quelle forme ces connaissances sont-elles organisées dans l'esprit et le cerveau humain pour permettre l'apprentissage et le fonctionnement effectif du langage ainsi que ses 
dysfonctionnements : s'agit-il de modules (encapsulés ou en interaction), ou bien les connaissances sontelles distribuées ? Quelle place accorder aux variations entre les langues face à l'universalité de la faculté de langage ? Enfin et surtout, une linguistique à visée cognitive ne saurait se désintéresser de la question des liens entre le langage et d'autres facultés humaines, également caractéristiques du fonctionnement symbolique de l'esprit, notamment des liens entre le langage et la pensée. Quelle est la nature du processus de représentation qui sous-tend l'activité de langage? Est-ce un processus spécifique, ou bien présente-t-il des analogies avec d'autres types de processus cognitifs (par exemple avec la vision)? Comment chaque langue met-elle en oeuvre de façon particulière ce processus ? Toutes ces questions engagent la façon dont la linguistique conçoit son articulation aux autres disciplines des sciences cognitives, ainsi que la nature du paradigme épistémologique qu'elle partage avec celles-ci. Se préoccuper de cette articulation ne signifie pas pour autant sortir de la linguistique. Mais cela conduit, d'une part à accorder une attention toute particulière à la notion de 'représentation' mise en œuvre par et dans la langue, et d'autre part à instaurer de façon explicite, au sein du travail proprement linguistique, une problématique d'ordre épistémologique.

Ces questions ne sont pas nouvelles, et certaines d'entre elles avaient déjà été abordées par d'autres théories linguistiques, antérieurement (ou parallèlement) au tournant 'cognitif' — seul retenu par l'historiographie officielle. C'est notamment le cas, en Europe, de théories post-structuralistes telles la psychomécanique de Gustave Guillaume ou les théories de l'énonciation.

Certes, ces deux courants majeurs se distinguent l'un de l'autre sur un certain nombre de points, en particulier sur la place et le rôle qu'ils accordent aux faits énonciatifs (Valette, 2003a; Valette, 2006). Mais ils s'accordent à considérer la langue comme un système sui generis de représentation possédant une logique propre, qu'il s'agit de dégager sans chercher à plaquer a priori quelque formalisme extérieur, et partagent l'idée que la mise à jour des principes qui sous-tendent cette dynamique de construction du sens constitue l'objectif du linguiste. Par la place centrale qu'ils accordent ainsi à la notion de 'représentation', ils ouvrent la voie à une problématique plus générale de la cognition; et par leur mode de théorisation de cette notion, ils annoncent, d'une certaine façon, le paradigme constructiviste de la linguistique cognitive actuelle.

\section{Théorie psychomécanique et cognition}

L'originalité de l'entreprise guillaumienne — à savoir la recherche d'une mécanique psychique à l'œuvre dans la langue - est longtemps restée incomprise. Or cette élaboration d'une 'linguistique cinétique' fondée sur les mécanismes mentaux sous-jacents aux formes de la langue constitue, selon les termes de Valette (2003b), "une tentative pour articuler mentalisme et mécanisme dans une théorie linguistique homogène". A ce titre, elle ressortit indéniablement à des préoccupations d'ordre cognitif. De là à dire que "la recherche constante, par Guillaume, de mécanismes mentaux (d'où le nom de 'psychomécanique' qu'il a donné à son œuvre) est très largement une forme de linguistique cognitive” (Hewson, 1997 : viii), il n'y a qu'un pas.

La dénomination de 'psychomécanique' que Guillaume a donnée à sa théorie lui a valu, on le sait, d'âpres critiques de la part des linguistes de son époque. Derrière le terme 'psycho-', d'aucuns ont voulu voir des relents de psychologisme, de mentalisme ou d'idéalisme : en un mot, une projection sémantique intuitive, jugée incontrôlée et incontrôlable, à l'opposé de procédures réglées et contrôlables. Ces détracteurs n'ont pas manqué d'être, à leur tour, taxés de 'positivistes' par Guillaume. Quant au terme '-mécanique', en dépit de sa connotation cybernétique, il a également été mal reçu. Faute de percevoir les liens possibles entre la démarche linguistique et la science du mouvement et de l'équilibre des corps, les contemporains de Guillaume ont en effet méconnu sa quête du mouvement sous-jacent à la construction des représentations par et dans la langue. Quant à l'alliance des deux termes, elle a paru incongrue voire contradictoire. Par la suite, diverses réponses de fond à ces critiques ont été apportées par des représentants du courant psychomécanique: sur la question du psychologisme, voir par exemple Toussaint (1997) ou Valette (2003a), et sur la mécanique intuitionnelle et son inspiration phénoménologique, voir Bajric (2005). 


\subsection{Une problématique cognitive en germe}

Les grandes options théoriques de la psychomécanique peuvent être interprétées dans une perspective cognitive : voir Valette (2006) et Fuchs (2007), dont le développement qui suit reprend les grandes lignes.

Le point central est celui de l'articulation entre la langue et le discours : celui-ci conduit à son tour à la question des liens entre langage et pensée. Pour Guillaume, l'activité de langage engage en effet deux moments théoriques distincts : celui de la 'langue', puis celui du 'discours'. Le premier peut être caractérisé comme permanent, fini, collectif et subconscient, alors que le second se présente comme éphémère, infini, individuel et conscient (selon les termes de Hewson, 1997 : 8-9). La langue correspond au plan de la 'représentation' et le discours à celui de 'l'expression'. Une telle distinction serait le propre de l'homme - par différence avec le cri animal qui n'instaurerait pas de distance entre l'acte d'expression et l'acte de représentation (Valette, 2003a : 22). L'enjeu cognitif est évident : c'est au plan de la représentation par la langue que se situerait la 'pensée pensée', inscrite de façon déterministe et mécaniciste dans l'esprit humain, cependant que la 'pensée pensante' se jouerait au plan de l'expression construite en discours par le sujet parlant.

Dès 1929, Guillaume assignait à la linguistique la tâche de remonter des unités 'd'effet' (du discours) vers les unités de 'puissance' (de la langue), afin de retrouver les opérations mentales qui sous-tendent ces dernières: "La vraie réalité d'une forme, ce ne sont pas les effets de sens multiples et fugaces qui résultent de son emploi, mais l'opération de pensée, toujours la même, qui préside à sa définition dans l'esprit" (Temps et Verbe). L'étude de la langue engage la problématique centrale de la 'chronogenèse' et du 'temps opératif'; cette problématique a donné lieu à de nombreux développements et à des interprétations divergentes. Disons schématiquement, à la suite de Valette (2003a), qu'elle a conduit à deux types de lectures antinomiques. D'un côté, une lecture qui privilégie la notion 'd'image-temps', et selon laquelle la langue serait une 'théorie' qu'il s'agirait en quelque sorte de révéler. Dans cette perspective, les représentations constitueraient autant d'images mentales que le sujet pensant se donnerait de lui-même ou de son activité pensante, et les saisies sur les cinétismes autant de captures d'images en discours. De l'autre côté, une lecture qui privilégie la notion de 'temps opératif' et assimile les saisies à des arrêts au sein d'un déplacement de la matière. D'où une affinité avec la notion de 'simulation' en sciences cognitives.

L'apport original de cette approche de la langue réside dans sa conception dynamique de la représentation comme mouvement, et non comme l'assignation d'étiquettes statiques — que ce mouvement (qui constitue la condition et la forme même de la concevabilité) soit, par ailleurs, représenté comme un cinétisme relevant de la mécanique, ou comme une oscillation dynamique relevant du mouvement dialectique. D'où le schème connu sous le nom de 'tenseur binaire radical', qui va de l'universel au singulier (du large à l'étroit) et inversement, et qui est présenté par Guillaume comme "la condition même de puissance de l'esprit humain". C'est précisément cette idée d'un mouvement de pensée continu, constitutif de la signifiance intrinsèque des formes de la langue, qui fonde toute la construction théorique.

Sur ce mouvement de pensée, des coupes ('saisies' ou 'interceptions') sont effectuées en discours, d'où résultent des effets de sens variables, selon l'endroit où elles opèrent. L'idée-force est donc celle d'une articulation entre continu (le mouvement) et le discontinu (l'arrêt sur mouvement). Cette articulation permet en particulier de rendre compte tout à la fois de la diversité des effets de sens en contexte et de l'unicité sémantique d'une forme réputée polysémique. Se trouve ainsi reprise, de façon beaucoup plus subtile, l'hypothèse du structuralisme (une forme - un sens).

La question des liens entre le langage et la pensée est donc centrale pour la psychomécanique. Guillaume avait choisi de défendre l'idée selon laquelle la pensée serait indépendante du langage et se saisirait ellemême via la langue, révélant ainsi ses schèmes cognitifs. "La pensée reste indépendante, en principe, du langage, et celui-ci ne représente que la puissance qu'elle se donne de se saisir elle-même et en ellemême" (Guillaume, Leçons vol. 9: 38), ou encore : "Le langage est dans l'homme pensant, dans la pensée humaine, un ouvrage par elle construit, qui lui sert — c'en est le finalisme principal — à reconnaître en elle-même où elle en est de sa propre construction" (Guillaume, Leçons vol. 13 : 13). Par là même, Guillaume annonçait ce que certains neuropsychologues ont par la suite argumenté à l'encontre 
des tenants d'un strict 'localisationnisme', à savoir l'idée que c'est l'ensemble du cerveau qui pense et qui raisonne, et que le langage constitue le moyen qui permet à la pensée de se penser elle-même.

\subsection{Guillaume et l'émergence des sciences cognitives}

La théorie de Guillaume n'est pas sans évoquer l'approche de la cybernétique, qui avait marqué, on le sait, la toute première époque des sciences cognitives. (Pour une présentation de la cybernétique, voir Varela, 1988 et Dupuy, 1994). Dès le tournant des années 1940 en effet, c'est-à-dire avant l'émergence du programme 'cognitiviste', les Macy Conferences avaient réuni les pères fondateurs (von Neumann, Wiener, Turing, McCulloch), pour tenter d'instaurer une nouvelle 'science de l'esprit'. Celle-ci devait s'appuyer notamment sur les disciplines formelles suivantes : la logique mathématique (pour décrire le fonctionnement $\mathrm{du}$ raisonnement), la théorie des systèmes (pour formuler les principes généraux gouvernant tout système complexe) et la théorie de l'information (comme théorie statistique du signal et des canaux de communication). L'hypothèse sous-jacente étant que la pensée fonctionnerait comme un calcul, à l'instar d'une machine (de là procèdera, plus tard, l'invention de l'ordinateur, selon les principes de von Neumann). Et c'est du côté de la physique (et non de l'algèbre ou de la biologie, comme le fera ultérieurement le cognitivisme) que les cybernéticiens allaient chercher leurs modèles, ce qui permettra ensuite l'émergence des théories de 'l'auto-organisation' - dans lesquelles la forme s'abstrait de la matière - et les approches du vivant comme 'propriété émergente du désordre'.

Guillaume a connu la cybernétique et a été - au moins pendant un temps — séduit par elle (Valette, 2003a : 17sq.). Son objectif initial, en élaborant la psychomécanique du langage, semble avoir été de construire une machine à penser, une sorte de cybernétique fondée sur le temps opératif et le tenseur binaire. A l'instar des cybernéticiens, il concevait la pensée comme asservie à la mécanique, et le langage comme constituant la partie mécanisable de la pensée. C'est pourquoi il entendait construire, en amont de la 'psycho-systématique' (ayant pour objet l'étude de la langue), une 'mécanique intuitionnelle' consacrée à l'étude des mécanismes psychiques qui président à la construction même des systèmes linguistiques et conditionnent leur structure : voir, sur ce point, l'Essai de mécanique intuitionnelle I (publié par Lowe, 2007 ) et le compte-rendu qu'en donne Guimier (sous presse). Selon Guillaume, cette mécanique — dont il entendait proposer "une analyse rigoureusement scientifique" (Essai : 144) — reposait sur la nécessité de penser par contrastes : le contraste univers/homme se reflétant dans le contraste universel/singulier, fondement du 'tenseur binaire radical' considéré comme l'opérateur général de structuration du langage.

En définitive, la perspective dans laquelle Guillaume a conduit son entreprise théorique n'est pas sans évoquer certaines recherches actuelles conduites au sein du paradigme dit 'constructiviste'. A l'instar de Rastier (1993) qui tient Guillaume pour "l'aïeul tutélaire de la linguistique cognitive à la française", on peut en effet voir dans les théories inspirées de René Thom (théorie des 'catastrophes', approches topologico-dynamiques) des "légataires du système de pensée de Guillaume". La psychomécanique constituerait ainsi une 'linguistique pré-cognitive', de type constructiviste — et peut-être même, plus précisément, du sous-type 'enactiviste', pour reprendre le terme de Varela (1988). (Si l'on suit Varela, l'histoire des sciences cognitives se compose en effet de quatre étapes successives. Viennent d'abord les jeunes années, marquées par la cybernétique. Puis c'est l'avènement des symboles avec le cognitivisme. Les deux étapes suivantes — le 'subsymbolisme', puis 1'“enactivisme' — participent du constructivisme : la cognition y est conçue comme une activité consistant à faire émerger dynamiquement des significations et non pas à traiter ou à réfléchir des représentations pré-existantes). Or si l'on admet que la psychomécanique comporte une dimension phénoménologique, alors il est loisible d'y voir une anticipation d'une certaine forme d'enactivisme. Quoi qu'il en soit de ce dernier point, l'Essai de mécanique intuitionnelle I contient certains passages consacrés à la genèse des modes de représentation de l'espace et du temps (pp. 92-103) que ne désavouerait pas un tenant des grammaires cognitives actuelles: le temps, non directement représentable, y est décrit comme empruntant à l'espace les conditions de sa représentation. 


\subsection{Vers une psychomécanique cognitive ?}

Au plan des grands principes théoriques qui la sous-tendent, la psychomécanique manifeste incontestablement des préoccupations d'ordre cognitif. Pour autant, l'assimiler purement et simplement à une théorie de linguistique cognitive constituerait, non seulement un anachronisme, mais aussi un contresens (à plus d'un titre).

Rappelons tout d'abord, qu'il manque une chronologisation des écrits de Guillaume, pour pouvoir véritablement mettre en perspective sa théorie au regard des développements actuels de la linguistique cognitive. Rappelons également que la comparaison est rendue difficile par la diversité des directions dans lesquelles la psychomécanique s'est déployée après Guillaume : à défaut d'une unification des approches se réclamant de la psychomécanique, un minimum de clarification conceptuelle et de stabilisation terminologique serait bienvenue.

Mais, par-delà ces considérations d'ordre général, revenons à la question de l'articulation possible de la théorie psychomécanique avec les sciences cognitives, et en particulier avec la neuro-psychologie. Guillaume lui-même ne s'était pas désintéressé des questions de pathologie du langage : Valette (2006) rappelle, par exemple, qu'il avait rencontré André Ombredane.

La voie d'un rapprochement possible semble ouverte par la notion de 'temps opératif', dont Guillaume postulait la réalité mentale effective. Certes, il estimait que les schèmes cognitifs de la pensée révélés au travers du langage doivent être étudiés par la linguistique, et non par la psychologie; mais cette précaution méthodologique n'interdit pas de penser que la réalité du mécanisme mental, ainsi mis à jour par le linguiste, pourrait être corroborée ensuite par la neuro-psychologie. Car si l'on prend au sérieux l'idée que le temps opératif correspond à des opérations mentales effectives, alors ces opérations devraient laisser des traces observables au plan comportemental et avoir un corrélat au plan cérébral. Tel était, du moins, l'espoir de certains psychomécaniciens de la première heure, tels Roch Valin ou Charles Bouton - espoir qui fut vite déçu : il ne se trouve plus guère à l'heure actuelle de psychomécaniciens pour tenter de construire une théorie 'neuro-compatible'(à l'exception de Maurice Toussaint, engagé dans une 'neurolinguistique épistémique' d'inspiration psychomécanique, affine avec la théorie des formes sémantiques et la théorie des systèmes dynamiques complexes).

Toutefois, une autre piste de rapprochement possible est évoquée par Monneret (1996, et 2003). Selon lui, au lieu de chercher à valider expérimentalement les concepts de la psychomécanique, on pourrait se demander, à l'inverse, ce que cette théorie serait susceptible d'apporter à la neuropsychologie. Celle-ci ne peut expérimenter que sur des productions, elle a donc besoin de théories qui articulent discours et langue ; par ailleurs, la prise en compte de la variabilité des productions langagières l'oblige à se tourner vers des modèles dynamiques. Or la psychomécanique répond à ces deux types d'exigences. Il serait donc intéressant de regarder si les outils théoriques qu'elle a développés permettent de rendre compte de certaines observations faites par la neuropsychologie. Ainsi, par exemple, dans le domaine de la pathologie du langage, les divers phénomènes apparemment disparates observés chez les agrammatiques pourraient-ils être éclairés grâce à certains concepts de la psychomécanique, comme le suggère Monneret. En effet, dans ce type particulier d'aphasie de Broca, les altérations de type omission semblent porter sur des éléments que la psychomécanique qualifierait 'd'avant', cependant que le remplacement d'un élément problématique semble opérer à l'aide de l'élément 'd'après' du système. Une telle piste mériterait sans conteste d'être explorée plus avant.

\section{Théories de l'énonciation et cognition}

Certains auteurs ont tenté de concilier psychomécanique et théories de l'énonciation, en proposant "une approche psychomécanique de l'énonciation" - pour reprendre le titre d'un article de Joly \& Roulland (1980). Force est toutefois de constater que l'un des principaux points de divergence entre ces deux 
courants concerne précisément la façon dont ils conçoivent l'articulation de la langue et du discours, c'est-à-dire leur approche des faits énonciatifs. Pour Guillaume, le mot passe du statut d'unité de puissance (en langue) à celui d'unité d'effet (en discours), grâce aux 'coupes' opérées sur le mouvement. Pour les tenants de l'énonciation, les unités se trouvent 'actualisées' à travers les opérations constructrices de l'énoncé, qui mêlent indissociablement prédication et référenciation : selon la formule de Benveniste (1964, repris dans 1966 : 131), "nihil est in lingua quod non prius fuerit in oratione".

\subsection{Aux sources de l'énonciation}

Après la clôture sur la 'langue' instaurée par Saussure, on a pu assister, dès le début du XX $\mathrm{X}^{\mathrm{e}}$ siècle chez les précurseurs de l'énonciation, à diverses tentatives pour renouer avec une problématique de la 'parole'.

Charles Bally s'efforçait d'articuler l'étude du système de la langue et une approche énonciative : grâce à la notion d'actualisation, il entendait "faire passer la langue dans la parole" $\left(1932,1944^{2}\right)$. Pour lui, toute phrase communique une pensée, c'est-à-dire une réaction subjective à une représentation objective ; elle comporte donc, dans sa structure sémantique, une partie 'modus' (constituée d'un sujet modal et d'un verbe modal) et une partie 'dictum'. L'acte fondamental de la communication, qui constitue l'acte d'énonciation complet, consiste à dire quelque chose ('propos') de quelque chose ('thème'). Cette structure binaire se retrouve, non seulement à l'intérieur de chaque phrase, mais aussi entre les phrases (reliées par une 'coordination sémantique').

En introduisant la catégorie des 'shifters' (ou 'embrayeurs'), Otto Jespersen et Roman Jakobson ont ensuite déplacé la problématique : d'acte individuel de production, l'énonciation devient trace du sujet dans l'énoncé. Cette approche a par la suite été développée et étendue par Emile Benveniste, qui a véritablement ouvert la voie d'une approche énonciative de la langue. Loin d'être des unités isolées, les 'indiciels' constituent, selon lui, un véritable système: les marques de personne, de temps, et de localisation spatiale permettent d'ancrer l'énoncé par rapport à la situation d'énonciation. L'énonciation est donc "directement responsable de certaines classes de signes qu'elle promeut littéralement à l'existence" (Benveniste, 1970, repris dans 1974 : 79-88), permettant ainsi au locuteur de s'approprier la langue pour la convertir en discours et d'énoncer sa position de locuteur à travers cet ensemble de signes particuliers que constitue "l'appareil formel de l'énonciation".

Au cœur du dispositif de la construction signifiante se trouvent donc le sujet énonciateur et la situation d'énonciation. L'énoncé porte la trace (en plein ou en creux) des différents modes possibles de présence (ou de masquage) du sujet énonciateur face au contenu actualisé. D'où la possibilité, à partir de l'analyse des valeurs prises par les marqueurs énonciatifs (valeurs de modalité, de temps, de personne, ...), d'établir une typologie des registres énonciatifs : on connaît l'opposition, faite par Benveniste (1959, repris dans 1966: 237-250), entre le registre de 'l'énonciation historique' (défini comme "récit des évènements passés sans aucune intervention du locuteur; personne ne parle ici, les évènements semblent se raconter d'eux-mêmes") et le registre de 'l'énonciation de discours' (défini comme "énonciation supposant chez (le locuteur) l'intention d'influencer (l'auditeur) en quelque manière”).

A une linguistique des signes et des structures, est ainsi venue s'ajouter une linguistique de l'énonciation. Il ne s'agit plus de passer de la langue au discours, mais de retrouver au sein même de la langue les conditions de sa mise en fonctionnement discursif. Selon Benveniste (1964, repris dans 1966 : 119-131; et 1967, repris dans $1974: 215-238$ ), la 'langue' a en effet un double statut : d'une part, c'est un "système de signes", lieu du 'sémiotique'; d'autre part, c'est un "instrument de communication (dont l'expression est le discours"), lieu du 'sémantique'. Le passage de l'un à l'autre de ces niveaux s'opère avec la phrase, définie comme "unité prédicative" et "unité complète qui porte à la fois sens et référence : sens parce qu'elle est informée de signification, et référence parce qu'elle se réfère à une situation donnée”. 
A ces contributions programmatiques de Benveniste, Antoine Culioli a, de son côté, tenté de substituer une véritable théorie des opérations énonciatives.

\subsection{La théorie des opérations énonciatives de Culioli}

Culioli s'inscrit dans la lignée des énonciativistes, en reprenant à son compte l'idée que le langage n'est pas un code extérieur à l'humain ni un calcul formel neutre intériorisé par lui et que les conditions de la mise en fonctionnement du système sont bien inscrites dans le système lui-même. Pour autant, l'énonciation ne se laisse pas décrire dans le cadre d'un schéma de la communication réduit à un simple échange d'informations. L'énoncé est le lieu d'une co-construction du sens (par l'énonciateur et son coénonciateur) qui laisse place aux ratés de la communication : la labilité et la déformabilité du sens sont des conditions intrinsèques de fonctionnement de la langue.

Dès la fin des années 1960, Culioli critiquait la grammaire générative ("la difficulté centrale de la formalisation en linguistique ne réside ni dans la formalisation de systèmes algébriques syntaxiques, ni dans l'étude distributionnelle des combinaisons de mots-objets en correspondance ponctuelle avec la réalité extra-linguistique") et soulignait la nécessité, pour le linguiste, de "bricoler" ses outils de formalisation (1967, repris dans $1999: 29)$.

La spécificité de l'approche culiolienne de l'énonciation se trouve exposée par l'auteur lui-même dans son intervention intitulée "Rapport sur un rapport" (1980) à la table-ronde organisée par A. Joly sur "La psychomécanique et les théories de l'énonciation". Culioli y distingue, schématiquement, trois grands types d'approches de l'énonciation. Le premier entend l'énonciation comme réalisation dans une situation d'une proposition potentielle, c'est-à-dire comme passage d'une phrase-type à une phrase-occurrence (appelée énoncé) : d'où un intérêt porté aux questions de prise de parole, de présupposés, de référence, d'acte de langage, etc. Le second entend l'énonciation comme distance entre le sujet énonciateur et son énoncé : cela pourra tendre, notamment, vers l'étude des modulations socio-linguistiques ou vers la stylistique. C'est d'un troisième type que se réclame Culioli, qu'il caractérise comme "une entreprise théorique de fondation, qui (prend) à l'origine le problème de la constitution et du fonctionnement des systèmes de repérage énonciatifs" et qui élabore un système de représentation métalinguistique de la constitution des énoncés.

Ni acte d'appropriation de la langue par un sujet, ni transition de la langue au discours, l'énonciation est donc plutôt conçue comme un mécanisme de construction : aussi s'agit-il, pour le linguiste, de décrire les opérations constitutives de la signification des énoncés, et de les formaliser (au sens fort d'une modélisation visant à reproduire les mécanismes en jeu).

Dans le modèle qu'il propose, la notion de 'représentation' occupe une place centrale. Selon lui (Notes $d u$ séminaire de DEA 1983-1984:5-8; repris dans $1990: 21-24$ ), cette notion peut intervenir à trois niveaux différents, qu'il est essentiel de bien distinguer. Le premier niveau est celui des 'représentations mentales' : ce niveau de conceptualisation de la réalité n'est pas directement accessible, il ne peut être appréhendé qu'à partir des activités humaines, notamment de l'activité de langage. Le deuxième niveau est celui des 'représentations textuelles' : l'activité de langage est une activité de représentation qui met en jeu des opérations linguistiques, lesquelles ont des traces sous forme de marqueurs linguistiques; ce sont donc des 'représentations de représentations' (spécifiques à chaque système linguistique), que le linguiste va chercher à isoler et à observer. Mais il est impossible de remonter directement de ce niveau à celui des conceptualisations: il faut construire un troisième niveau, celui des 'représentations métalinguistiques'(système de termes primitifs, de règles et d'opérations), en espérant que le passage du niveau 2 au niveau 3 simule adéquatement celui du niveau 1 au niveau 2. Autrement dit, "il nous faut construire un système de représentation qui porte sur ce système de représentation qu'est la langue" $(1990: 23)$. 
A ce niveau métalinguistique, le linguiste doit pouvoir effectuer des calculs, dans lesquels opérations prédicatives (repérages entre les termes constitutifs de la relation prédicative) et opérations énonciatives (plongée dans un système de coordonnées spatio-temporelles dont le sujet de l'énonciation constitue le repère-origine) sont étroitement intriquées.

\subsection{De l'énonciation à la cognition}

Les approches énonciatives de la langue — très présentes dans les travaux de linguistique française — ont été développées, comme il a été dit plus haut, de façon totalement indépendante du tournant dit 'cognitif' de la linguistique d'Outre-Atlantique. Si le cognitivisme classique aborde le langage comme moyen d'expression de la pensée, les approches énonciatives préfèrent, quant à elles, mettre en avant (à l'instar $\mathrm{du}$ fonctionnalisme) la fonction de communication du langage. Par lui-même, un tel choix n'est, à l'évidence, nullement exclusif d'une ouverture à la cognition: on se souvient, à cet égard, des contributions de Jakobson, aussi bien sur les 'fonctions du langage' et le 'schéma de la communication' qu'en matière d'aphasie ou d'acquisition du langage.

L'insistance sur la dimension communicative du langage semble d'ailleurs indirectement confortée par certains acquis récents des neurosciences. Que l'on songe par exemple au fait que chez le singe l'aire comparable à l'aire de Broca (crucialement impliquée, chez l'homme, dans l'exercice du langage) est l'aire F5, où l'on a découvert les fameux 'neurones miroirs' essentiels pour l'imitation et la communication, et que chez l'humain l'aire de Broca enregistre, elle aussi, les gestes des personnes avec lesquelles il communique.

Par-delà ces considération d'ordre général, il convient de rappeler que la théorie des opérations énonciatives de Culioli a été élaborée dans un cadre résolument tourné vers la cognition. On évoquera ici les échanges interdisciplinaires suivis et réguliers que ce linguiste a instaurés durant de nombreuses années avec le psychologue François Bresson (lui-même inscrit dans la lignée théorique de Jean Piaget) et le logicien Jean-Blaise Grize, ainsi qu'avec des spécialistes de pathologie du langage (comme Jean Laplanche et André Bourguignon) dans le domaine de l'aphasie et de la schizophrénie.

Si cette familiarité avec les problématiques des autres disciplines des sciences cognitives a, sans conteste, inspiré l'élaboration de la théorie des opérations énonciatives, elle a également conduit Culioli à dénoncer les risques d'assimilations abusives liés à la notion de 'cognition' : “A term like 'cognition' shows itself to be dangerously ambiguous, for it is used to refer to mental activity, to simulation, to a whole series of unverified simplifications : of representational activity to neuronal activity, to give but one example" (1995:31).

A cet égard, la position de Culioli se démarque de celle des tenants des grammaires cognitives, pour qui il n'existe pas de différence de nature entre les représentations conceptuelles et les représentations sémantiques à l'œuvre dans les langues (voir par exemple Langacker, 2003). Pour ces auteurs en effet, les structures sémantiques des langues sont des conceptualisations effectuées à des fins symboliques, qui sélectionnent certaines facettes dans l'éventail des conceptualisations déployé par la pensée prélinguistique, et qui les organisent de façon variable : la spécificité du linguistique réside donc dans la sélection et la mise en forme de ce contenu conceptuel. Dans une telle perspective, la distinction culiolienne entre les trois niveaux de représentions se trouve obérée.

Il n'en reste pas moins que les schémas spatiaux proposés de façon assez intuitive par les grammaires cognitives rappellent certains opérateurs métalinguistiques de Culioli (en particulier le 'repérage' et ses différentes valeurs) : de façon générale, se trouve à l'oeuvre une même quête d'invariants du langage derrière les variations des systèmes linguistiques. Bien qu'étrangers l'un à l'autre, ces deux courants s'alimentent en effet à des sources d'inspiration 'constructiviste' : d'où, par exemple, leur commune recherche d'outils formels en direction de la topologie (très largement illustrée, chez Culioli, dans ses travaux sur le 'domaine notionnel' représenté grâce à un appareillage topologique impliquant un intérieur, 
un extérieur, une frontière et un gradient dans l'intérieur). L'analyse des points de convergence et de divergence entre la théorie des opérations énonciatives et les grammaires cognitives constituerait sans nul doute une étude en soi ; pour une première approche concernant leur commun "terreau continuiste", voir Fuchs \& Victorri (eds.) (1994).

En définitive, comment l'énonciation rejoint-elle la cognition? Le pari consiste à postuler que le passage entre les représentations textuelles et les représentations métalinguistiques simule de façon analogique le passage entre les représentations conceptuelles et les représentations textuelles; ou, pour le dire de façon imagée, que les (méta-)opérateurs du linguiste sont aux opérateurs de la langue ce que ceux-ci sont aux opérateurs de la pensée. Selon Valette (2006 : ch. 12), cet espoir de retrouver derrière les opérations énonciatives l'analogue d'opérations cognitives ne serait pas partagé par tous les adeptes de la théorie : aux 'cogniticiens' (tenants de la portée cognitive de l'hypothèse simulatoire) s'opposeraient les 'métalinguistes' (pour lesquels opérations énonciatives et opérations cognitives seraient constitutivement irréductibles les unes aux autres). Seules des expérimentations neuro-psychologiques (encore à venir) contribueraient, peut-être, à les départager. Culioli, pour sa part, semble esquiver la question, comme en témoigne cette déclaration malicieuse lors d'une intervention dans un symposium consacré au thème 'Langage et Cognition' : "the aim (of this paper) is not to answer basic questions, but rather to suggest what the questions are, leaving it to the audience to draw their conclusions as to whether I am a nativist, a maturationist, a constructivist, a cognitivist, a conceptualist or a Platonist, etc. etc., unless I am a mongrel in a class of my own" (1989; repris dans $1990: 177)$.

\section{Conclusion}

A l'issue de ce parcours, il apparaît que la linguistique française a bien été, tout au long du $\mathrm{XX}^{\mathrm{e}}$ siècle, un creuset où se sont élaborées des théories du langage originales tournées vers des problématiques cognitives - et ce, de façon indépendante de la linguistique dite 'cognitive' retenue par l'historiographie officielle.

L'avenir de ces théories reste à écrire. Leur ancrage effectif dans des recherches cognitives interdisciplinaires suppose que soit évité un double écueil, signalé par Lazard dans son article polémique évoqué plus haut: d'un côté, la perte de ce qui fonde la spécificité de leur démarche proprement linguistique ; et, de l'autre, l'affaiblissement des exigences formelles, au nom d'un effet de mode risquant de conduire à une dissolution de la problématique cognitive dans une sémantique "molle".

\section{Références bibliographiques}

Bajric, S. (2005). Questions d'intuition, Langue Française, 147, 7-18.

Bally, Ch. (1932, 1944²). Linguistique générale et linguistique française. Berne : Francke.

Benveniste, E. (1966). Problèmes de linguistique générale. Paris : Gallimard.

Benveniste, E. (1974). Problèmes de linguistique générale II. Paris : Gallimard.

Culioli, A. (1980). Rapport sur un rapport. In Joly, A. (ed.), La psychomécanique et les théories de l'énonciation, Lille : Presses universitaires, 37-47.

Culioli, A. (1990). Pour une linguistique de l'énonciation, vol. 1. Paris / Gap : Ophrys.

Culioli, A. (1995). Cognition and Representation in Linguistic Theory. Amsterdam/Philadelphia : Benjamins.

Culioli, A. (1999). Pour une linguistique de l'énonciation, vol. 2 et 3. Paris / Gap : Ophrys.

Dupuy, J-P. (1994, rééd. 1999). Aux origines des sciences cognitives. Paris : La Découverte.

Fuchs, C. (2004). Pour introduire à la linguistique cognitive. In Fuchs, C. (ed.), La linguistique cognitive, Paris : Ophrys/Maison des sciences de l'homme, 1-24. 
Fuchs, C. (2007). La psychomécanique est-elle une linguistique cognitive ? In Bres, J. \& al. (eds.), Psychomécanique du langage et linguistiques cognitives, Limoges : Lambert-Lucas, 1-53.

Fuchs, C. \& B. Victorri (eds.) (1994). Continuity in linguistic Semantics. Amsterdam / Philadelphia : Benjamins.

Guillaume, G. (1929). Temps et Verbe. Paris : Champion.

Guillaume, G. (1989). Leçons de Linguistique 1947-48 (vol. 9). Lille : Presses universitaires, et Québec : Presses de l'Université Laval.

Guillaume, G. (1995). Leçons de Linguistique 1958-59 et 1959-60 (vol. 13). Paris : Klincksieck, et Québec : Presses de l'Université Laval.

Guimier, Cl. (sous presse). Compte-rendu de R. Lowe (dir.) (2007). Bulletin de la Société de Linguistique de Paris (soumis).

Hewson, J. (1997). The Cognitive System of the French Verb. Amsterdam : Benjamins.

Joly, A. (ed.) (1980). La psychomécanique et les théories de l'énonciation, Lille : Presses universitaires.

Joly, A. \& D. Roulland (1980). Pour une approche psychomécanique de l'énonciation. In Joly, A. \& W. H. Hirtle (eds.), Langage et psychomécanique du langage : études dédiées à Roch Valin. Lille : Presses universitaires, et Québec : Presses de l'université Laval, 537-581. Repris en 1980 dans Joly, A. (ed.), Essais de systématique énonciative. Lille : Presses universitaires, 11-58.

Langacker, R. (2003). Grammaire, cognition et le problème de la relativité : le cas de la possession. In Vandeloise, Cl. (ed.), Langues et cognition. Paris : Hermès, 205-237.

Lazard, G. (2007). La linguistique cognitive n'existe pas. Bulletin de la Société de linguistique de Paris, CII :1, 3-16.

Lowe, R. (dir.) (2007). Essais et mémoires de Gustave Guillaume. Essai de mécanique intuitionnelle I. Espace et temps en pensée commune et dans les structures de langue. Québec : Presses de l’Université Laval.

Monneret, Ph. (1996). Pour une psychomécanique des pathologies du langage : étude de neurolinguistique théorique. Thèse, Université Paris IV.

Monneret, Ph. (2003). Les exigences théoriques d'une neurolinguistique guillaumienne. Le Français Moderne, LXXI : 1, 26-36.

Rastier, F. (1993). La sémantique cognitive: éléments d'histoire et d'épistémologie. Histoire, Epistémologie, langage, $X X: 1,133-146$.

Rouveret, A. (2004). Grammaire formelle et cognition linguistique. In Fuchs, C. (ed.), La linguistique cognitive, Paris : Ophrys/Maison des sciences de l'homme, 27-71.

Toussaint, M. (1997). Le sujet du temps. Cahiers de Praxématique, 29, 185-203.

Valette, M. (2003a). Enonciation et cognition : deux termes in absentia pour des notions omniprésentes dans l'œuvre de Guillaume. Le Français Moderne, LXXI : 1, 6-25.

Valette, M. (2003b). Intentionnalité du sujet et téléonomie de la langue dans la linguistique cognitive / énonciative. In Ouattara, A. (ed.), Parcours énonciatifs et parcours interprétatifs : théories et applications. Gap / Paris : Ophrys, 289-301.

Valette, M. (2006). Linguistiques énonciatives et cognitives françaises: Gustave Guillaume, Bernard Pottier, Maurice Toussaint, Antoine Culioli. Paris : Honoré Champion.

Varela, F. (1988, rééd. 1996). Invitation aux sciences cognitives. Paris : Le Seuil. 